\title{
Categories and Preferences Among Category Systems
}

\author{
Dale Jacquette*
}

\begin{abstract}
Category systems are explained and defined. Historical examples are discussed in relation to the concept of a taxonomy in which one category subsumes another subcategory. The task of category selection and arrangement is characterized as the work of conceptual analysis of the same body of facts or relations. Category system building is further described as a process of identifying commonalities at the appropriate level of conceptual abstraction and analysis. An inverted tree-like diagramming convention is developed to exhibit category system relations. Formal principles are introduced to explain requirements for comparative economy of distinct category systems as a preference basis, involving numbers of Links and Nodes in category system taxonomies. Conflicting and incommensurable preference rankings and their implications are considered.
\end{abstract}

\section{CATEGORIES}

Categories are general concepts, the most general there could be. Category systems are supposed to reflect basic conceptual divisions and branching but always one-way subsumption relations between categories as distinct kinds of things. Categories can be local rather than global, concerning a limited choice of general properties restricted to a subset of ontic relations in the greater categorical scheme, for special focus and attention. Any true or false taxonomy of plants or animals satisfies the description. If we designate the Link relation between category and subsumed subcategory with an arrow $\rightarrow$, then one Node $\mathbf{O}$ linking a choice of two biological categories can be depicted as, Mammal $\mathbf{O} \rightarrow \mathbf{O}$ Placental. Or, equivalently, and in complicated applications more informatively, two-dimensionally rather than linearly:

Mammal<smiles>OO</smiles>

Placental

Diagram 1.

* University of Bern, Switzerland

(c) The Author 2015. Published by Oxford University Press on behalf of The Hegeler Institute.

All rights reserved. For permissions, please e-mail: journals.permissions@oup.com 
Mammals are positioned above Placentals, with the arrow pointing from Mammal category to Placental category, in this graphic glossary, to represent the ontic dependence relation that explains subsumption, whereby if there were no Placentals, there could still be Mammals, whereas if there were no Mammals, then Placentals could not exist. The beginnings of more elaborate configurations of categories in elementary applied category systems is indicated by extension of the zoological taxonomy in a further step, dividing up subcategories of two major kinds of mammals:

Mammal

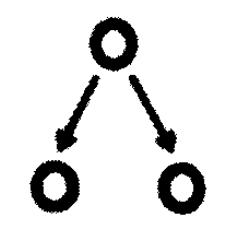

\section{Placental Marsupial}

\section{Diagram 2.}

The two basic (lateral and multiple branching) forms, instantiated in Diagram 1 and Diagram 2, are all we need to understand the combinatorics of category systems representable as branching tree-like structures. This includes at least all scientific taxonomies that can be built out of the two forms, and all ontic metaphysical category systems, as found in the philosophical literature of general ontology, reproduced and discussed further below. From every category Node we either lateral drop to another category Node or we branch into two or more subsumed category Nodes.

Category systems are inventive human creations that serve a pragmatic end, when they do, but attract no philosophical interest when they are not, positively supported by the facts of the world. There are two steps that must be followed, the same in both cases, in order to arrive at constructive category systems like those instantiated in Diagram 1 and Diagram 2. After useful commonalities are first abstracted, recognized in different specimens, genera, and so on, then category systems are called upon at some stage of or coextensive with the process of category system building to organize and spatially arrange tokens representing whatever commonalities have been chosen for systematization. Even when the facts-based commonalities themselves do not budge, what is made of them, how they are classified and interrelated with other facts, interpreted, conceptualized, understood, and, indeed, categorized, whether they are recognized and included or excluded from consideration, makes an enormous difference from category system to category system, as each proposes to explain the most basic metaphysical concepts implied by a correct understanding of the world from a different philosophical perspective.

There is no higher reason than scientific prudence why the biological category Fish should not encompass all permanent life-cycle swimming water animals, in which case seals are excluded but whales and cetaceans generally are categorized as special kinds of fish. Thankfully, biologists have insisted on good scientific grounds that even lifetime environment and mode of locomotion are accidental rather than essential to their subsumption-relation location on a madly branching animal 
taxonomy, some parts only of which are not blurry and conjectural. The ideal in biological taxonomies is to have one-way subsumption relations exactly correspond to one-way lines of evolutionary descent. Does this mean that if modern human beings (Cro-Magnons, Homo sapiens sapiens) are evolutionary descendents of Neandertals (rather than the latter being an evolutionary offshoot), that Neandertals subsume modern humans in the evolutionary biological taxonomical category system? It does in fact, if to subsume as explained means that if a subsumed category did not exist then the subsuming category could still exist, but not conversely. If the subsuming category did not exist, then the subsumed category could not exist. If modern humans are evolutionary descendents of Neandertals, then modern humans are subsumed by Neandertals in the same sense that if modern humans did not exist, Neandertals could still exist, but not conversely. If Neandertals did not exist, then modern humans, supposing them to be descendents of Neandertals, could also not exist. It would be no different than you or I existing despite the nonexistence at any time or place of any of our effective progenitor germ-plasm donors.

Whether or not a category system is correct, by which is meant without unanswerable objection and preferable on explanatorily relevant grounds to its competitors, can therefore be determined in part by its proper recognition of categoryarranging relevant facts. The process of category system building also requires a philosophically circumspect abstraction of relevant commonalities, the exact choice of categories made for the category system under construction, and how they are best judged to be interrelated. Category system building, to state only the obvious, is an artifact of human decision, convenience, conceptual utility, expectations for adequate expression of what are deemed the most basic relevant kinds of things, opinions, and efforts to justify beliefs that these expectations are satisfied. Explanatorily unruly ultimately subjective quasiaesthetic factors and criteria, and explanatory pragmatics, among related considerations, also enter into the actual choices made when confronting a category system diagram, like those presented in what follows. If such choices for category systems were always made in the same way, then that fact would be a natural sign that the harmonious category systems were fundamentally correct.

The disappointing truth of the matter is that, from the pre-Socratic beginnings of metaphysics and all throughout its history to the present day, there have been multiple category systems, championed by individual thinkers and their schools. Each correspond to different metaphysics and ontologies, supported by and supporting different epistemologies and philosophical methodologies, that in the extreme are mutually logically inconsistent. Category systems are diverse in many often incommensurable ways. Like mathematical notations, languages, artworks, and artifacts of all kinds, scientific and philosophical theories, including category systems, are cultural objects.

Categories are not themselves natural things to be found on the beach or by digging in the earth. Interesting, then, is it not, that we choose to represent category systems with categories as though they were growing on and from other categories in a tree? As though categories naturally after all grew on trees. When all along of course the natural order of categories is due to a reasonable ordering of what expert human 
thought finds satisfying. Whales are not fish, but water mammals. Whales are fish with lungs. We decide what categories there are, and if we are scientific, then we undertake efforts at category system building that positively reflect what we believe to be the existent order of things. That (all) placentals are mammals and not the other way around, for example. We must identify and study these animals in order to understand how their respective categories are interrelated.

Category systems like other artifacts of human ingenuity are things that are made for a cluster of purposes, and independently of their originating objective cannot be properly understood. Among the unlimitedly many categorical systems that might be devised, some will inevitably be preferred over others, just as philosophical concepts, distinctions, arguments, theories, and applications have their energetic proponents and opponents. It is possible, in consequence, to understand category systems as two- or three-dimensional schematic or tabular representations in and of ontology, exhibiting typically inverted tree-like dendritic or branching form. To posit that all being can be divided into Substance, Essence and Accident, along roughly Aristotelian lines, is to advance an ontology or substance theory ousiology, with its main subdivisions depicted by a forking bifurcation.

\section{CRITERIA FOR COMPETING CATEGORY SYSTEMS}

The question whether a given category system is preferable to its competitors in the sense of being uniquely correct is no different in principle than the question whether a corresponding discursive theoretical ontology (including substance-theory ousiology) or metaphysical system of existent entities is uniquely correct. The reader can perform a phenomenological experiment by visually inspecting the diagrams including category labels for alternative category systems, alternative ontologies or ultimate metaphysics of the most general kinds of things, and all that they subsume, and noting which of them seem more satisfying, complete in category choice, and properly arranged.

Metaphysical systems are sometimes judged explanatorily incomplete if they do not include an ontology of metaphysical categories. Categories are kinds of being or existence in such applications, so that ontology as the metaphysics of being is generally interested in precisely these categories of existence, of what general kinds of things exist, and how they are ontically and conceptually interrelated. The theoretical alternatives are usually extreme, as illustrated by the ontic controversy as to whether properties are sets, or sets properties. The question can be understood as asking whether sets subsume properties, or properties subsume sets, in a correct category system that includes as categories Sets and Properties. Or, alternatively, among many similar kinds of polarities, whether propositions are logically possible worlds, or vice versa. After all, it is equally thinkable to interpret logically possible worlds conversely as an enormously long conjunction of descriptive propositions that is itself a single truth functionally complex proposition, than it is to think of propositions as states of affairs existing in some or all logically possible worlds. And so on. Every interesting 
conflict in metaphysics can be interpreted as a choice between alternative category systems with different categories or different subsumption relations.

The choices are reminiscent of those encountered in theoretical geometry, where it is possible to define lines as the shortest connection of points between any two points, and equally acceptable to define points as the intersections of any two lines. Whether points are ontically more basic and subsumptive than lines, or the reverse, is entirely a matter of category-system preference, for which there can be rational grounds. Nor is it decisive for the interdefinability of these basic geometrical concepts that intuitively Euclidean points are 'smaller' than lines, and that Euclidean lines are lines made up of as though presupposing densely compacted Euclidean points. The definitions objectively understood can go either way, and we do not propose to dictate mathematical truth from the standpoint of whatever relation may be most comforting or cognitively agreeable to commonsense expectations. The burden of developing an adequate ontic category system, in the controversy over fundamental ontology, must then include such divisions of prioritization as those that make propositions ontically more fundamental than worlds, or conversely. Controversies are not quelled, because others are certain to prefer the advantages of characterizing sets as ontically more fundamental than properties, or the opposite. Among countless possibilities. Similar problems of preference apply for all the same reasons to category schemes. The effect when we consider the entire field of metaphysics is not to see a single tree spreading forth its branches, but an eclectic orchard of many different kinds of trees, and many differently configured roots, branches, and vines linking together the categories of being (or Being, if we mean to signal something grand thereby, by capitalizing what is already referred to by a singular term).

Each distinct category system represents an opportunity to restructure our understanding of the metaphysical relations holding among the most basic categories of being in different ways. Is there substance? Are there substances? What does substance categorically subsume? Some candidate categories, combinatorially speaking, may be nonstarters, destined to be dismissed by sound adjudication virtually out of the box on grounds of implausibility, unworkability, or lack of coherence with better established, more deeply entrenched philosophical commitments with which certain ontologies of categories may be logically incompatible. Others may proliferate like memes at a cocktail party in the right sort of philosophical culture, attaining the deeply entrenched status of being a virtually unquestionable starting-place for all relevantly related inquiry, and a rock on which theory might build.

When categories are arranged in a two- or three-dimensional branching display, the relationship represented when a subcategory label is placed immediately in relation to and typically beneath another category label is, as previously mentioned, subsumption of the first designated category by the second. Thus, the category Mammal in the animal taxonomy example subsumes the abstracted category Placental, placentals being a more specific kind of mammal, in exactly the specific extracted part of the taxonomic branch relating Mammal $\mathbf{O} \rightarrow \mathbf{O}$ Placental. The category Primary Substance in the higher metaphysics of an Aristotelian ousiology or substance 
ontology subsumes Essence and Accident, or the like. Placed at the ends of a forklike branching configuration, the subsumed categories are graphically illustrated as spreading away from and not subsuming or being subsumed by one another. Although other further and finer subcategorical subdivisions may be subsumed by each of these in turn, as when the subcategories Space, Time, and Relation, with further subcategories especially of Relation, or the like, are shown or said in the category system to be subsumed as more specific subcategories of Accident. All of these illustrations are subject in principle to overthrow by competing category systems in promoting a different ontology. Why speak of substances or (Primary) Substance at all?

\section{CATEGORY DIAGRAMMING SAMPLER}

The importance of category system diagrams in explaining categories and their corresponding metaphysical ontological distinctions and interrelations among the kinds of things a theory says exists is illustrated by Roderick M. Chisholm on the first page of the 'Introduction' to his A Realistic Theory of Categories: An Essay on Ontology. There he explains the concept of a category (among the "ultimate categories of reality") by producing an inverted branching tree-like diagram of category names linked together by slanting mostly bifurcating lines:

This book is about the ultimate categories of reality. It is about categories, not about theories of categories. What a category is may be shown by depicting the table of categories that is defended here.

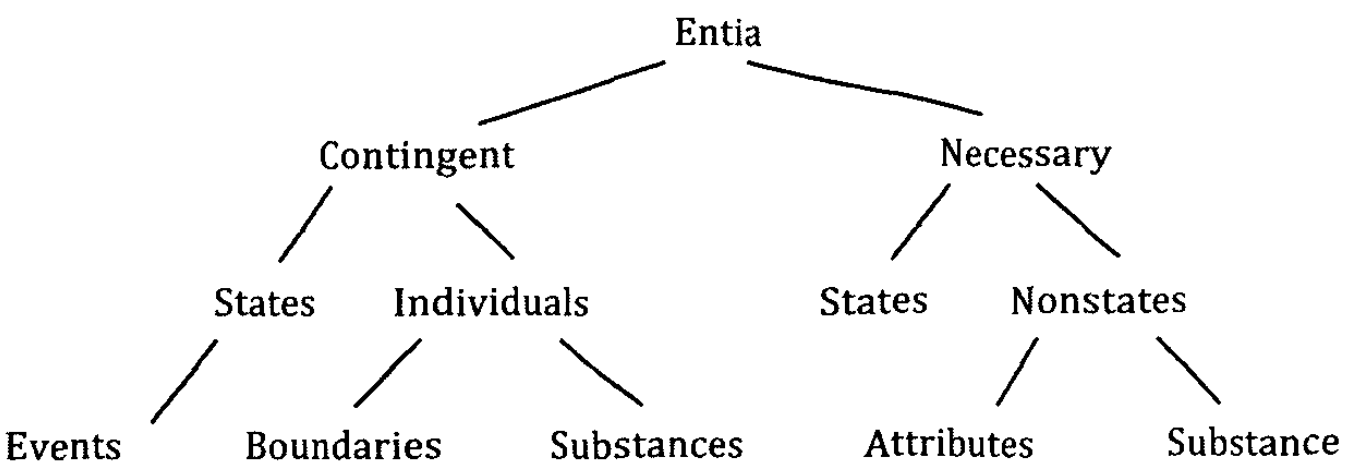

Diagram 3. A Table of Categories

Franz Brentano, in his 1862 dissertation, Von der mannigfachen Bedeutung des Seienden nach Aristoteles (On the Manifold Senses of Being in Aristotle), offers after lengthy scholarly discussion the following arguably flawed reconstruction of his own explication of what he takes to be Aristotle's category system. After explaining that being itself cannot be a category of being for Aristotle, Brentano presents a category system branching diagram as the summary and final statement of his detailed investigations of Aristotle's writings on the categories, considered through the lens of Brentano's thorough scouring of the secondary classical and philosophical literature. ${ }^{2}$ 


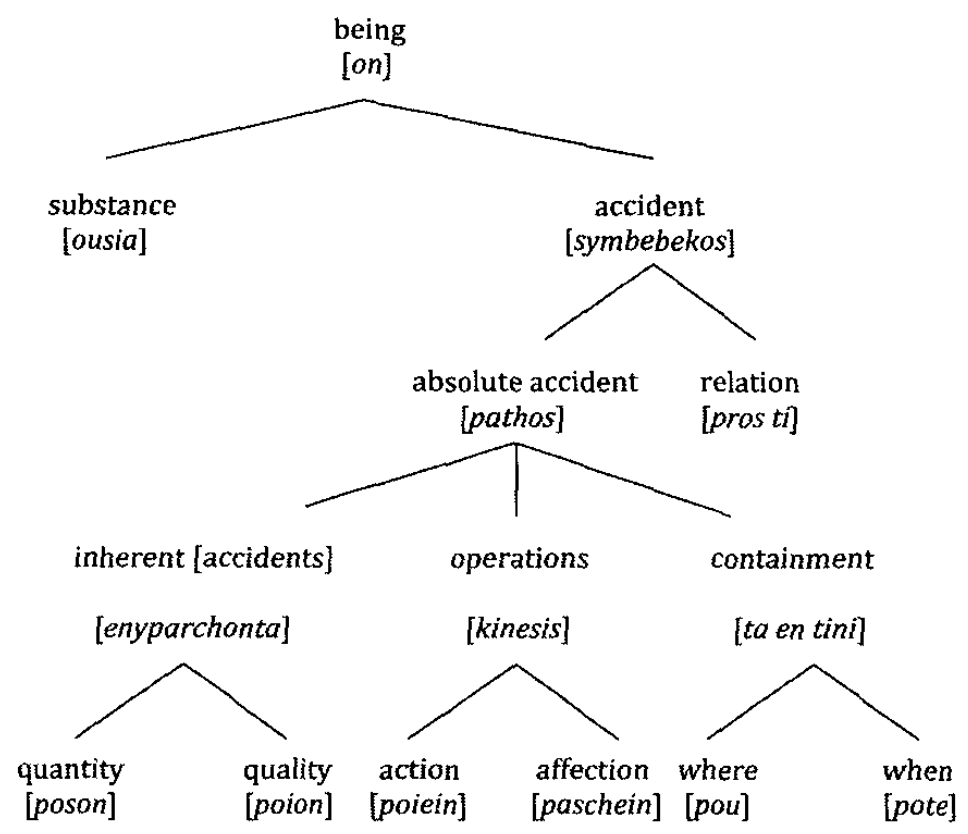

Diagram 4. Brentano's 1862 Dissertation Interpretive Diagram of Aristotelian Categories

An alternative branching depiction of Aristotle's category system that is arguably more in keeping with Brentano's interpretation of Aristotle than Brentano's own category diagram is illustrated on the basis of Brentano's historical-philosophical scholarship as having this simplified structure: ${ }^{3}$

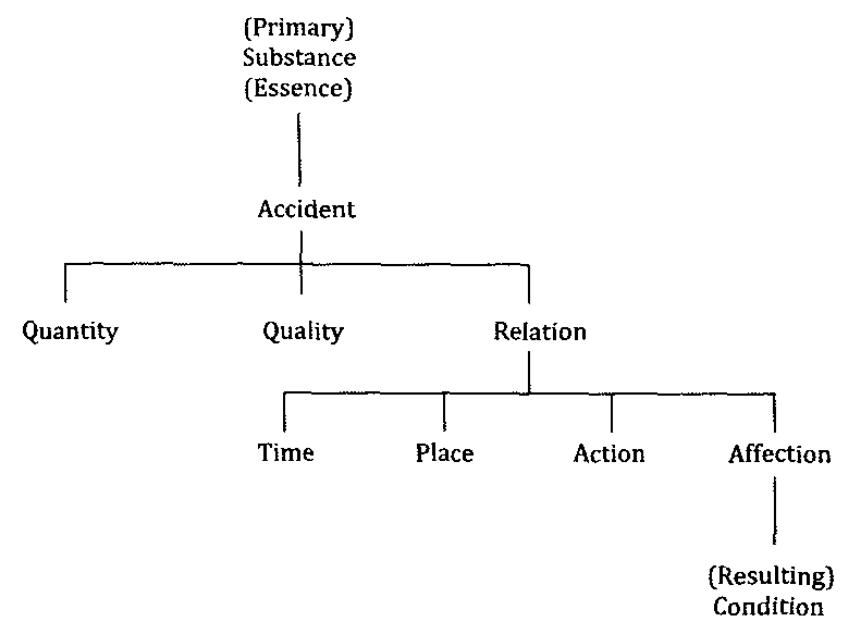

Diagram 5. Aristotle's First Philosophy Fundamental Categories of Being (Multiple Predicative Senses of Being)

The schematization of category systems in any field of application in a table or tree of categories can be a useful instrument, not merely for presenting the results of taxonomic investigation, but for purposes of inquiry. Like a logical or mathematical notation, category trees show us at a glance how general concepts might be thought to stand in relation to one another. This representation of basic categories inevitably raises interesting metaphysical questions at the same time that it provides a framework and model for understanding many kinds of categorical subsumptions.

Prior to theoretical ontic commitment, the same technique enables the investigator to experiment with alternative arrangements. Diagrams permit judgment to test 
out visually the intuitive sense of categorical subsumptions on paper, to move their tokens around on the board until the right combination, the one that seems most correct, is achieved, when everything seems to snap into place. As happens in different ways with different resulting category systems differentially preferred by different category system builders. Whether or not we can more easily understand how categories and subcategories are related in a category system by graphic means, pictorial representation in a branching tree diagram provides a valuable supplemental tool for grasping categorical subsumptions in an area of fundamental ontology where we can use all the help we can get. Category system diagrams facilitate reasoning in ways analogous to those by which we more readily discern the underlying inference structure of a deductive argument by casting it in symbolic logic, or sketch a diagram for an engineering project. As we can visually understand different colored subway routes represented in a Harry Beck structural diagram from 1931 geometrically simplifying the actual routes for the sake of displaying their stops and connections. ${ }^{4} \mathrm{We}$ benefit in performing arithmetical computations from a fully expressive abstract mathematical notation, with an algorithm bank for specific calculations, using Arabic rather than Roman numerals, and all the machinery of elementary mathematics, rather than relying on ordinary colloquial language.

The streamlining of categorical systems is seen in the reconstruction of Brentano's Kategorienlehre diagram, devised by Brentano's editor, Alfred Kastil, in the 1933 edition of Brentano's later writings on ontology. Brentano, if Kastil has rightly understood Brentano's intent, arrives at a neo-Aristotelian table of ultimate ontic categories that is different in structure, much more simplified, than the table of categories Brentano attributes to Aristotle in his dissertation (Diagram 4), and even than the proposed revision that is arguably more faithful to Brentano's verbal description of Aristotle's theory of categories (Diagram 5). Kastil hazards this branching tree of Brentano's category system in the frontispiece to Part Three of the Kategorienlehre, 'The Final Three Drafts of the Theory of Categories' from 1916:'

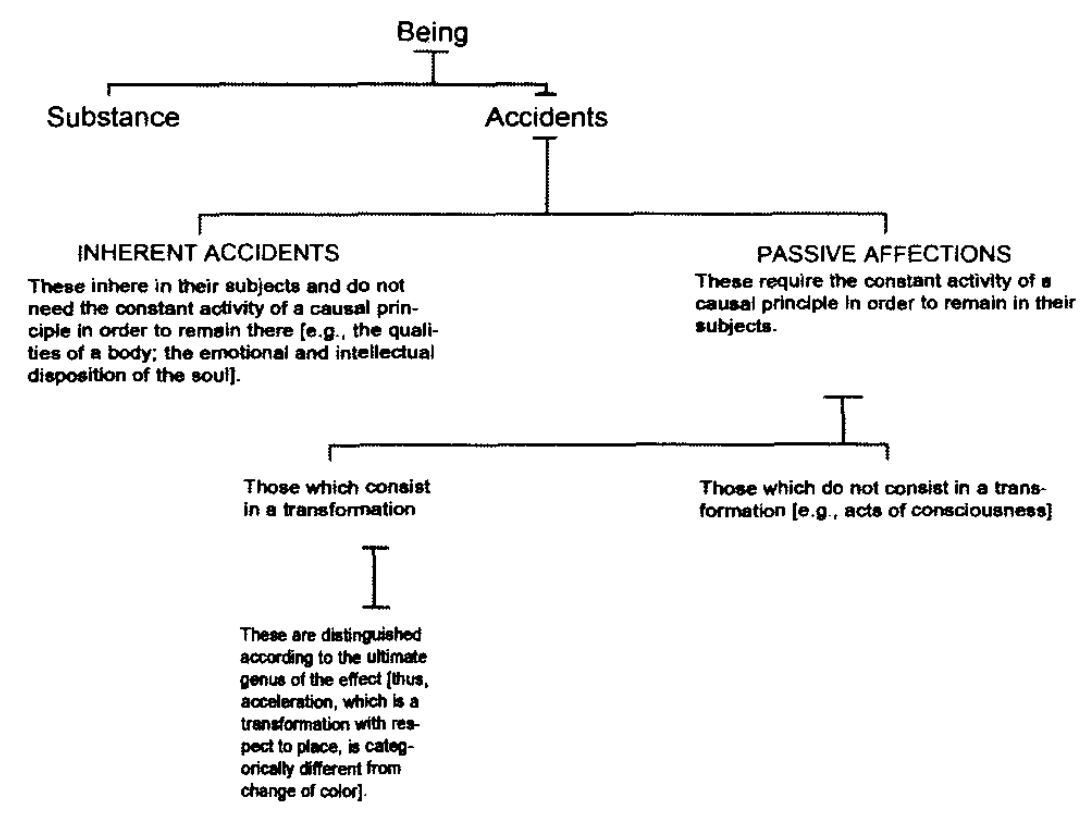

Diagram 6. Brentano's (Later neo-Aristotelian) Table of Categories (reconstructed by Kastil for the Kategorienlehre) 
The potential cognitive advantages of category system trees are undisputed. How category systems should be depicted is often the main point of metaphysical controversy. Diagrams for category systems must correspondingly graphically include Nodes and Links, as seen in all branching tree diagrams depicting a category system. The question as to what Nodes a category system includes is the question what categories of things the system is designed to systematize. How the chosen categories are linked together we have argued is by subsumption relations, by one category subsuming another. Subsumption we explain as being such that if the subsuming category did not exist, then the subsumed category could not exist, but not conversely. If the subsumed category did not exist, it would still be possible in principle for the subsuming category to exist.

In every category system, the tasks are therefore nothing less than those expected of the most complete and thorough conceptual analyses of any related body of facts, from among which those resulting in preferable category systems are often the most basic, universal, and metaphysically insightful. Philosophy needs to determine what general categories of things there are, and how they are interrelated, whether by subsumption or nonsubsumption, which is to say by mutual conceptual (extensional) independence and exclusion relations. We need to know what kinds of things are supposed to exist in a theory's referential domain of discourse, and what kinds of things belong to more general types of other kinds of things. With this information in hand, discursively or diagrammatically, graphically, pictorially, or by any other expressive vehicle, everything minimally needed for a complete category system, irrespective of its preferred method of exposition, is fully available, and the work of devising a system of categories is accomplished. All of which, needless to say, is easier said than done.

What further directions and philosophical advice can responsibly be offered those who admire the tidy exhibit of categories and subsumption relations in an area of conceptual analysis afforded by a branching tree diagram, neatly labeled with the types of things that are supposed to stand in such an such metaphysical relation to one another by virtue of possessing either a one-way subsumption relation or mutual exclusion from immediate subsumption of one another on distant branches or parts of branches? Category system building requires the abstraction and arrangement of commonalities, following an exact selection of relevant facts as abstraction basis. Theorists must decide, among predetermined types of things, which of one type are items (they could be universals) to be included in or excluded from the items or individuals of another distinct type (substances). All dogs are mammals, not the other way around. All mammals are vertebrates, also not the other way around. There is no royal road to attaining these judgments. We have to roll up our sleeves, proceed by intuition in a spirit of conjecture and hypothesis, undertake scientific investigations or profit second-hand from expert scientific knowledge, and be prepared to make adjustments in the network of analyzed concepts within which we are searching for a definite order of subsumption dependencies. It is these interrelations that we expect to shed light on every component and the greater entity they may constitute as a whole reality in metaphysics, in every specialized application of category-system structures. It includes any that explains the nature of existence as a function of 
the structural comprehension and internal interconnections of the basic categories presupposed by, explicit or implied in, a body of discourse taken as a subject of inquiry.

Category systems, like any other substantive contentful contributions to philosophy and science, are challenged and need to be defended against objections from a variety of quarters. Relying on the biological taxonomy analogy again, a classification of animal types by which cetaceans are subsumed in the more general category of water-dwelling zoomorphs, along with fish, is destined to be replaced by a preferred taxonomy in which the structural analogues among a selection of animal types is made the basis for subsumption of whales as kinds of cetaceans, and cetaceans as kinds of mammals, in a subsumption hierarchy as it has come to be developed, refined, and accepted in the natural biological sciences, regardless of where the animals in question happen to be environed and what evolutionary history has produced their animal taxonomy-categorized individuals.

Whales and dogs have lungs; fish do not. Whales, accordingly, like dogs, together by virtue of other essential similarities, are mammals, and not fish, even though whales like fish live in the water. Why? Because biologists, most prominently Carl Linnaeus (1707-1778), have decided that, for scientific purposes, having lungs, among other structural biological commonalities, is more important than environment or mode of locomotion, most general types of animals having niched in all environments and mastered every type of moving from place to place on earth, in water and air. ${ }^{6}$

The point is that in designing category schemes for any area of interest, we must be attuned to essential similarities and analogies, as well as differences, among a spectrum of species and specimens, and to the subsumption relations by which one kind of thing under consideration belongs to, is contained in, or subsumed by another. Mammals subsume marsupials, and not the other way around. Taxonomies of living things are as much branching tree-structured subsumptions in a variety of combinatorial arrangements as are the metaphysical category systems of Aristotle, Brentano, and Chisholm, among many others. Like those artifactual scientific taxonomies developed for purposes of scientific inquiry and explanation, fundamental ontological category systems involve an unpredetermined choice of basic categories to be arranged in one-directional subsumption relations that is nevertheless constrained by the facts from which abstractions of basic categories are made and then linked together in branching subsumption trees. (In German, a whale is still correctly denoted both $W a l$ and Walfisch today, while in contemporary Dutch, the same category of animal is generally denoted only Walvis. Which is not meant to imply that Nederlands speakers today do not understand that whales are not fish.)

Philosophers spend much of their time and energy on precisely such questions of category subsumption versus exclusion. To choose one example of which the history of philosophy is replete, doxastic voluntarists like Descartes argue effectively that belief states are subsumed in a category of voluntary psychological events in the 
philosophy of mind as mental acts for which the thinker is responsible. Thus, according to Meditations on First Philosophy IV, it is unchecked will that is responsible for the mind's sometimes arriving at false beliefs, while doxastic involuntarists like David Hume staunchly deny any such subsumption in a taxonomy of mental event categories. Unless or until such philosophical disputes are resolved, if such a thing should turn out to be possible, there can be no settled agreement on the relevant metaphysical category and subcategory system for this corner of the ontology as it relates to the nature of belief and the possibility of exercising willful control over the content of beliefs. Then the two trees, one Cartesian, in which beliefs are subsumed as voluntary mental act events, and another Humean, in which beliefs are not subsumed as voluntary mental act events, must continue to exist awkwardly side by side as rival ontologies. Some ontologies predictably will be championed by adherents who vigorously criticize opposing arrangements, whereas the banners of others will be taken up by another group of defenders. As philosophical dialectic in the application historically unfolds in the conflicting concentrated category systems of Hume and Descartes, alternative options are promoted on both sides, motivated by criticisms of competing category systems in the marketplace of ideas. Category-system tree diagramming expedites this process by showing visually and unequivocally what categories are chosen for each system, and exactly how the categories they include are supposed to be subsumption interrelated.

To appreciate the cognitive utility in what is at stake among competing category systems, consider by analogy whether Neandertals belong to the same evolutionary branch or only a sidebranch as Homo sapiens sapiens. The relation, only one of which at most can be true, is pictured in different ways as different trees or selected branches of evolutionary bushes. The scientific interpretation of admitted bioarchaeological evidence has not yet stabilized, from what a layman learns on-line, and DNA analysis, always reconstructive first in the Neandertal case, may be equivocal. I say 'may' because I am not an expert, merely a consumer of this information. The only point is to show that evolutionary anthropology has at different times been offered a choice theoretically between a model of modern human development that includes Neandertal on its own immediate evolutionary tree, and another model that excludes Neandertals from the human family. One learns again from popularly available sources that reconstruction of DNA sequences is generally required even in forensic applications involving recent crimes, suggesting with appropriate qualifications again that it may be even more probably indispensable in the case of long extinct hominids.

Suppose that Neandertals and Sapiens were capable of interbreeding, but not as frequently producing fertile offspring. Maybe Neandertals would only mate in the springtime, and Sapiens were more flexible in their receptivity. Or the like. We need only the breath of a story. Then we have these alternative models of Neandertal-Sapiens taxonomic category systems. The left-hand model represents modern human beings as descendants of Neandertal, and the other depicts them as distinct species, as the long slanted rectangle in the diagram is meant to show, that might have genetically 
interacted, albeit, perhaps without long-term evolutionarily success. The pictures offer two competing direct and side branch ontologies of evolutionary descent:

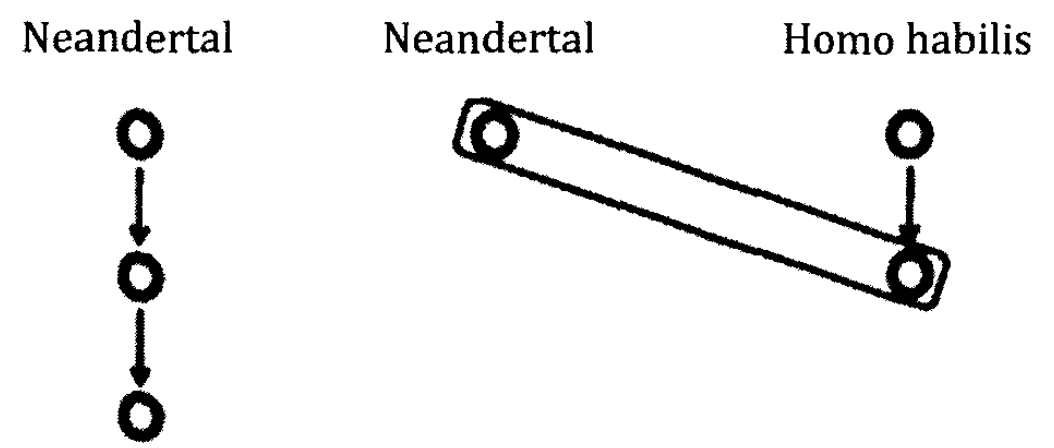

Sapiens

Sapiens

Diagram 7.

Just as it makes an important difference in biology and evolutionary anthropology whether humans are or are not part of the descent of Neandertals, so in philosophy it is vital to determine correspondingly whether Substance is included in or excluded from the kinds of Being in a metaphysical category system. It is central to Aristotle, but is altogether excluded from Being in Plato's metaphysical category system, where Substance means what it does for Aristotle, but Being is very differently understood as eternal and unchanging, transcending the ephemeral phenomenal world of Becoming.

Diagramming of category systems is a strictly inessential, but highly useful practical technique for discovering and presenting categories and their interconnections with subcategories. The simplicity and elegance of a category system as graphically represented, when we are offered a look at a picture of the categories and how they are supposed to interrelate, can itself constitute an argument in support of the corresponding metaphysical interrelationships that a philosophical theory may approve. When the relationships in a category system are more complicated than we would have thought or preferred, then we are sometimes confronted with the possibility of reducing a category system's complexity level in favor of a simpler or more economical reorganization of categories in a competing more attractive image of categories and their subsumptions. There is not agreement universally among metaphysicians on a single category system, but a plethora of alternative competing metaphysical category systems.

\section{NODES AND LINKS AND LEVELS OF CATEGORY-SYSTEM TREE DIAGRAMS}

What principles, if any, govern the choice between alternative competing category systems? Multiple reasonable answers might naturally be tendered to so loaded a philosophical question. Among the decisive quasiaesthetic criteria for preference of one category system over another, one branching tree diagram of categories and 
nestings of the subcategories they subsume, as more appealing than certain of its competitors, we concentrate for the moment on the comparative economy of entities explicitly mentioned in alternative category frameworks, and turn thereafter to their comparative simplicity.

What is and what should be meant by 'economy' among competing category systems? What does the criterion mean, and what does it amount to? These are questions that can only be adequately answered by a precise formal theory of the comparative dimensions of alternative category systems. The theory must make provision for assigning a metric of relative values to category systems integrated into a network of applied logical principles to relate the comparative measurements of distinct category systems. These in every case are alternative arrangements of a choice of categories that either differently combine the same categories as its competitor category systems, or provide all necessary ontic explanations and metaphysical grounding of the same relevant concepts and applications as its competitors, but in a more economical way.

What is wanted is a rigorous choice of principles that define the relevant dimensions of category systems for purposes of comparison of their absolute dimensions, the absolute cardinality of category-system elements and structural components, and finally their variable preferability, at least in relation to questions of relative categorysystem economy. We begin with basic concepts by which to assemble a framework for discussing the parts and their relative more or less economical choice and organization of categories. We do not insist that category systems only be compared in case they meet all the same explanatory expectations. Different category systems are often developed precisely to flaunt or out of frustration concerning the limitations of ruling metaphysical conventions, and should be considered as having no specific formal structural obligations beyond that of explaining what kinds of most basic things exist and how those kinds of things are ontically related.

For simplicity, the topology and parameters of two-dimensional branching category systems only are considered. We imagine the inverted dendritic tree structures of categories and subcategories, subsubcategories, and so on-not indefinitely, perhaps, but in principle for a sufficiently long run in the case of interesting kinds of relationships of genus, species, subspecies, and so on, under any appropriate nomenclature, in order to exhibit worthwhile features of the most basic ontic categories. We generally expect fundamental metaphysics to involve more limited abstracted categories and subsumptions of subcategories by categories, in a simpler picture, as we like our natural laws of physics to be relatively elementary in formulation, involving in this case the most basic categories of being. We focus on branching tree categorical diagrams, on the assumption that whatever we can say about pictures of categories we can also say about tabular and discursive descriptions. Branching diagrams of categories by popular acclaim are generally considered more intuitive, and the extension from two-dimensional representation to three is trivial, should such a need be perceived. On all counts, two-dimensional branching depictions of category relations are undoubtedly the best place to start. A category system branching tree, regardless of which specific configuration is preferred, will need to make provision for every kind of existent thing that is required to properly classify and arrange all 
the facts in the theory of being's purview. The strategic question is always what exactly is actually needed, why is it needed and in what sense?

A category system, as previously mentioned, consists of category Nodes and connecting Links. Nodes represent, and are typically labeled in category-system diagrams in order to explicitly designate, specific categories of things. Nodes appear in relation to one another only by virtue of being graphically connected together by means of Links. What the Links represent and whether there is more than one kind of Link between Nodes is entirely the business of each individual category system and what and how it proposes to picture the ontology it champions. We lay down principles for the measurement of overall comparative size of different category systems as represented by tree-structured branching diagrams of category interrelations in several dimensions, so that we can make judgments of comparative economy, first, and then simplicity or complexity. We work toward definitions of the depth and width of entire tree-branching category structures, as a simple sum of the depth and width of individual branches considered exhaustively in the tree structure, and as contributing exclusively if at all to either the tree's total depth or width, exclusively.

These values then provide the key to justifying judgments of comparative economy and ultimately, ceteris paribus, of comparative preferability. At least we shall have a measure of how the systems compare in that specific respect, supposing them to be otherwise explanatorily competitive. Nodes $\mathbf{O}$ and Links $\rightarrow$ are shown in the diagrams presented for consideration below respectively as small circles and connecting descending arrows from one horizontal plane in the branching diagram to the next. All Links descend from a higher to a lower level in the branching diagram, representing that what is higher subsumes what is below. Lateral links of configuration $\mathbf{O}-\mathbf{O}$ are not considered or allowed. Whenever we might be tempted to suppose that there are mutually unrelated separate links of Links between Nodes, we may consider them as involving an implicit higher category, as in the explications. Here the horizontal double arrow is meant to show that were the apparently separate originating Nodes in a category-system diagram on the left, we can assume that there is some more general category subsuming them both and restoring unitary origin to the category system. Here we picture the transition from implicit to explicit unitary origin of a category-system tree, from two starting arrangements:

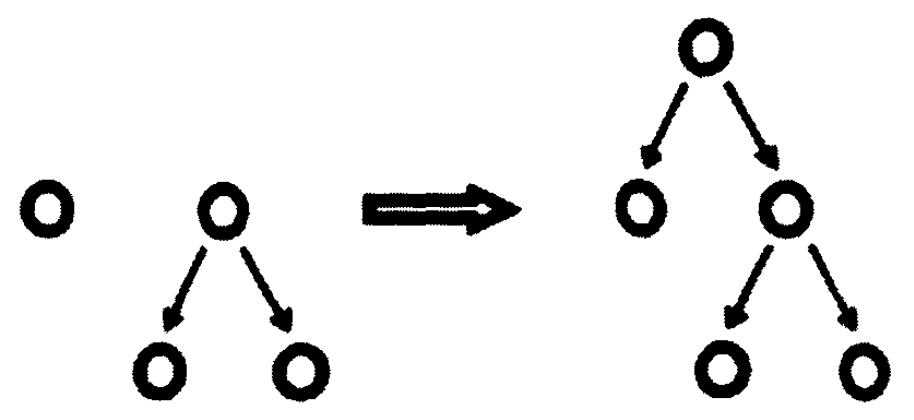

Diagram 8. 


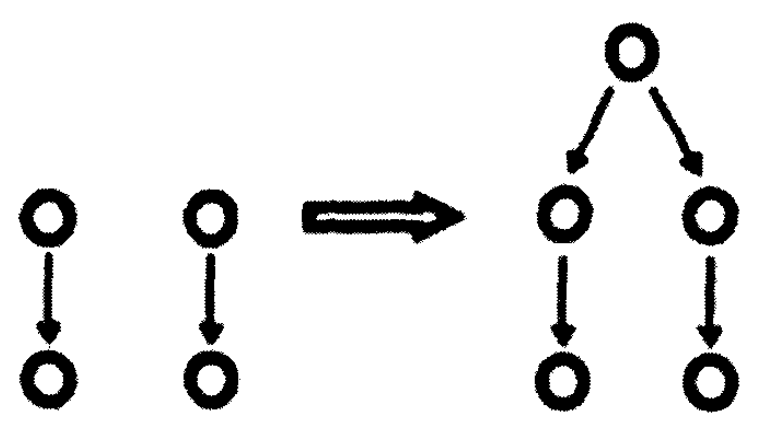

Diagram 9.

Can this always be done? The intuitive rationale for supposing that such an expansion of implicit origin for what otherwise appear to be separate unconnected category subsumptions in a category system is that we can generally identify a single thing to which the two category subsumptions are supposed to belong, even if the origin is chosen so generally, as we find in Brentano's Diagram 4 and Diagram 6, to refer only to 'Being' or 'What Exists' or another general subject of the categorical subsumptions systematized and graphically depicted.

Links connecting Nodes in category-system trees can have forms like, but not limited numerically to, the following:
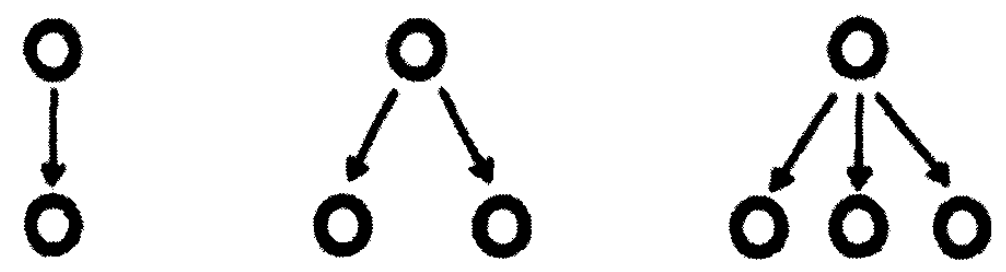

Diagrams 10, 11, and 12.

Subsumption is generally graphically represented on the simplified conventions adopted here as vertical drop of Nodes, reinforced by the direction of arrow-headed Links. What is depicted below is understood as subsumed by what is connected by arrows to what is depicted above, subsuming whatever appears connected vertically below it in the diagram.

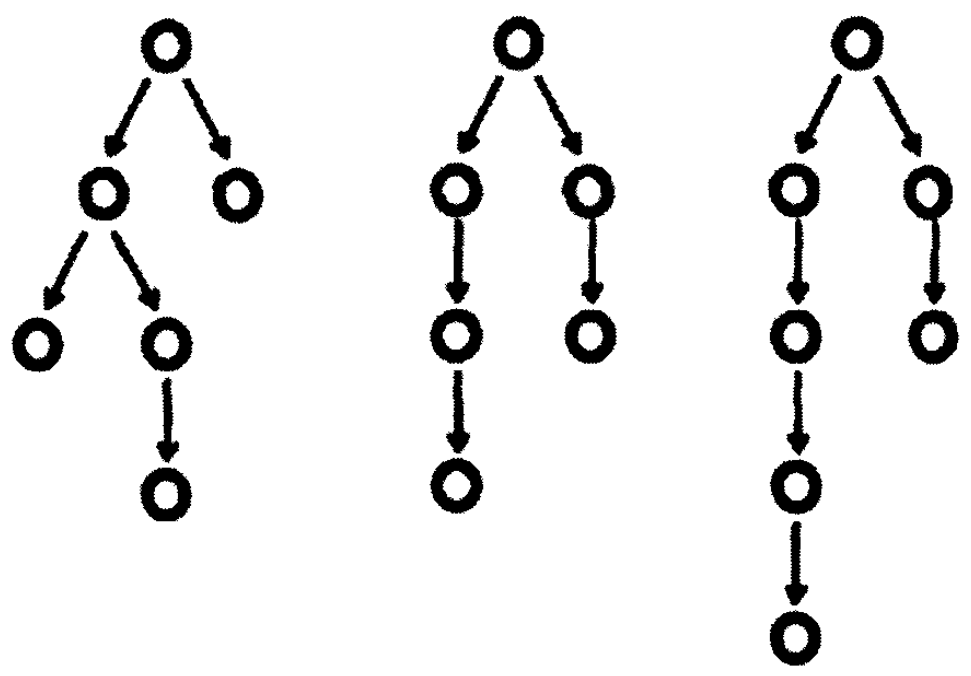

Diagrams 13, 14, and 15. 
Here we encounter a first sense of the potentially conflicting measures of economy and simplicity among category systems. Where category systems are depicted graphically above as Diagram 13 has precisely as many category Nodes (six) and Links (five) as that shown in Diagram 14. Although in some sense, because Diagram 13 contains three branches rather than two, like that of Diagram 14 and Diagram 15, the category system depicted by Diagram 13 seems more complex than that of Diagram 15, whereas the category system of Diagram 15 contains more components, in total seven Nodes and six Links.

We refer to the cardinality (number or size) of the Nodes and Links in a category system CS as Card(Nodes/Links(CSN)) $=n$. Generally, if $n$ Nodes are eliminated from a CS, then $n$ Links are eliminated in the process; while overall, for 2-dimensional branching category-system diagrams, $\operatorname{Card}($ Nodes $)=n \rightarrow \operatorname{Card}($ Links $)=n-1$. An Index of the economy of a CS, Index $(E c o n(C S))$, is ideally wanted, whereby we can compare it with that of others, including all competitors, to establish first the relative economy of the category systems, and, on its shoulders, the relative (ceteris paribus) theoretical preferability of one category system over others on grounds of relative economy, should any advantaged category system emerge in comparative evaluation of these quasiaesthetic characteristics.

\section{ONTOLOGICAL ECONOMY AND SIMPLICITY PARAMETERS FOR CATEGORY SYSTEMS}

We have developed a concept of relative category-system simplicity or complexity by adding up the number of category Nodes whenever there are more than 2 at any level from all levels in the category-system branching diagram evaluated for economy and simplicity. Width of a category spread in the tree trumps length alone in complexity determinations, no matter how long the run of categories without branching a category-system branching diagram contains. Although comparative economy is not always the most decisive factor in preferential evaluations of competing category systems, we want to be able at the end of inquiry to justifiably affirm a choice based on number of Nodes as economy preference ePref ${ }^{>}$:

(P1) $\forall C S_{m, n}\left[\operatorname{Econ}^{>}\left(\mathrm{CS}_{m}, \mathrm{CS}_{n}\right) \leftrightarrow \operatorname{Card}\left(\operatorname{Nodes}\left(\mathrm{CS}_{m}\right)\right)<\operatorname{Card}\left(\operatorname{Nodes}\left(\mathrm{CS}_{n}\right)\right)\right]$

(P2) $\forall C S_{m, n}\left[E \operatorname{Econ}^{>}\left(C S_{m}, C S_{n}\right) \rightarrow e \operatorname{Pref}^{>}\left(C S_{m}, C S_{n}\right)\right]$

We could rewrite (P1) as $\left(\mathrm{P}^{*}\right)$, substituting 'Links' for 'Nodes'. (P2) is something of an explanation of how ePref ${ }^{>}$is meant to be understood. There are other grounds than economy for preferring one category system over another. Principle (P2) identifies only category-system economy as a specific, explicitly designated rationale for theoretical preference.

The interesting question in any case is not the comparative economy of competing category systems, but how to determine the simplicity versus complexity indexes of alternative category systems. We simply add up the Nodes or Links of competing category systems as they would be represented by branching tree diagrams in order to determine their relative economy and preferability on grounds of economy. 
Simplicity or complexity is another, more complex, matter. If we cannot simply add up and compare the total numbers of Nodes or Links of competing category systems in order to decide which if any is structurally more complex, then what standard of relative simplicity is appropriate?

As a first step in this direction, we distinguish between the levels of categories in a category system tree. The reference is meant literally, where a category system is represented by means of an inverted tree, with the originating category Node at the top and all other lower category Nodes so graphically diagrammed as related by means of Links to the main and top Node. From top to bottom, there are usually successive levels of spreading and receding connections of Nodes, as the category system is articulated in the form of a branching tree. If we were to draw it out from top to bottom, it would appear to grow like an upside-down dendrite, as distinctions are recognized and kinds of things are shown as ontically related to other kinds of things. There is always a certain number of Nodes, usually one, appearing as the main or top Node in a complete tree. It is then followed in descent at specific levels, separated ideally in each instance by exactly the length of a Link. There are usually other Nodes below the first level, connected to the Nodes above them by Links, and so on as the tree branches out. We adopt the convention of using arrows to indicate the subsumption of subordinate (point directed downward) by superordinate categories in the tree. The obvious levels of the category system are demarcated in this stratification:

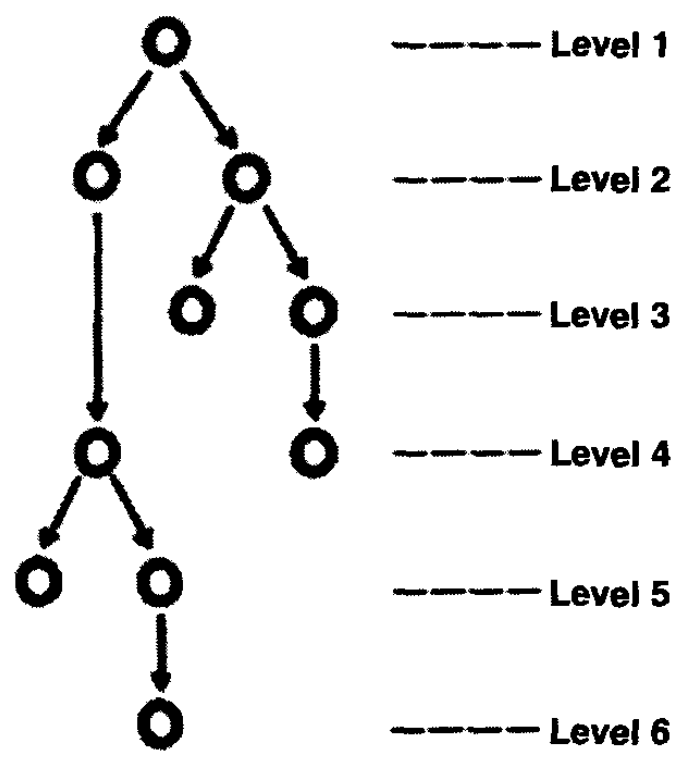

Diagram 16.

To determine the measure of simplicity (equivalently, complexity) of a category system N, Index (Simp (CSN)), the total number of Nodes greater than 1 occurring at each level $n$ in the tree are added together. The expression for the relation adopted 
here is this, relying again on the number of Nodes at different Node levels as standard:

(P3) $\forall C S_{m}\left[\operatorname{Index}\left(\operatorname{Simp}\left(C_{m}\right)\right)=\operatorname{Card}\left(\operatorname{Nodes}>1\left(\forall L_{n}\left(C S_{m}\right)\right)\right)+\operatorname{Card}\left(\operatorname{Nodes}\left(C S_{m}\right)\right)\right]$

Here the right hand side can alternatively be expressed as :

$$
\sum_{n=1}^{i}\left(\operatorname{Card}\left(\operatorname{Nodes}\left(L_{i}\left(C S_{m}\right)>1\right)\right)\right)+\operatorname{Card}\left(\operatorname{Nodes}\left(C_{m}\right)\right)
$$

An index of simplicity (equivalently, complexity) serves something like the purpose of the cardinality of Nodes or Links as an index of economy. We count the number of Nodes occurring at each level in a category system diagram, adding them together in order to obtain a measure of category system simplicity (complexity). We observe, negatively, first of all, that Card(Nodes $(C S)+\operatorname{Card}(\operatorname{Links}(C S)) \neq$ Index(Simp $(C S))$. We cannot decide that one category system is more complex than another, assuming (usually unrealistically) all the same explanatory burdens, merely by counting the Nodes and Links in each system. If further illustration is wanted, the fact is plainly seen in the following category-system diagrams:

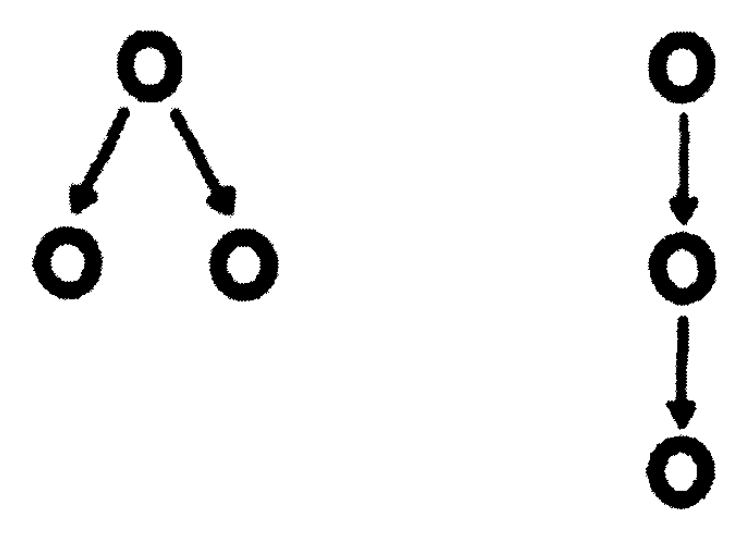

Diagram 9, Diagram 17.

The category system shown in Diagram 9 has precisely the same number of Nodes (three) and Links (two) as Diagram 17, although in an obvious sense Diagram 9 represents a more complex category system than Diagram 17. Complexity and economy among category systems is not merely a matter of the cardinality of Nodes and Links, but in some sense of the width of category systems, which is to say of how widely they branch. This is measured by summing up the number of Nodes appearing at each distinct level, as a criterion of how widely the category system is spread into increasingly more fine-grained subsumptions. For this purpose, it is not sufficient merely to count the number of branches. We identify a counterexample visually in the following comparison of category system trees, where the category system represented by Diagram 18 is manifestly more complex than that depicted by further recycling of Diagram 9, even though they both contain the same number of branches (two).

Where comparative complexity is concerned, we cannot simply indiscriminately count the numbers of bridges or Links that split (Splinks) in two or three or more subdivisions, because we immediately encounter such counterexamples as represented in 
the following opposition of system diagrams. Rather we tally up the total number of Nodes plus the total number of Nodes at each level of the CS at which more than 1 Node occurs. When comparing the category system represented by Diagram 9 and Diagram 18, we arrive in the case of Diagram $9 C S$ at $3+2=5$ as simplicity (or complexity) index, and for the CS of Diagram 18, at a total of $7+2=9$, satisfying the pretheoretical intuition that the CS depicted by Diagram 18 is more complex than that of Diagram 9, on the grounds that its simplicity index is higher:
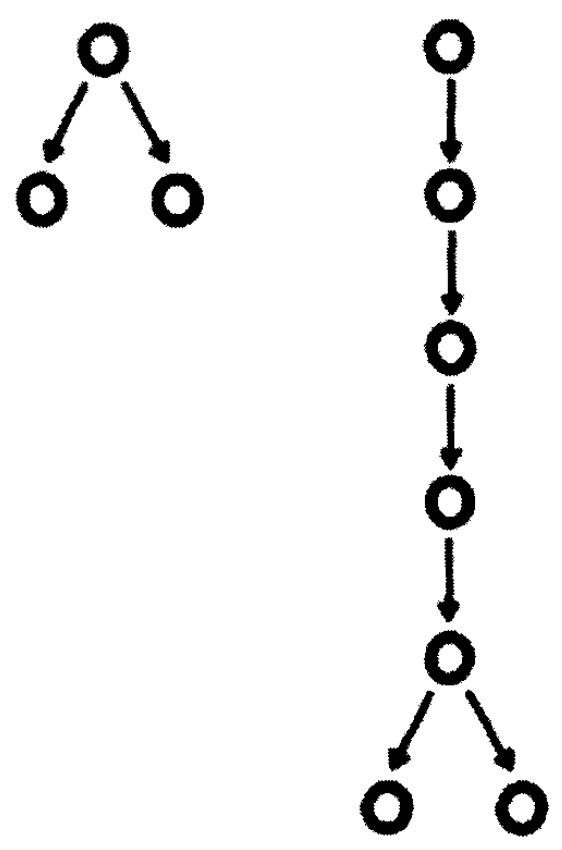

Diagram 9, Diagram 18.

Similarly, in the case of these two graphic diagrammings of category systems, represented by Diagrams 19 and 20 . We see precisely the same number of branching configurations, 2 in each category system, whereas their simplicity indexes reflect the obviously greater complexity of the category system shown in Diagram 20 as compared with that of Diagram 19. The simplicity index of the CS represented by Diagram 19 is $2+2+5=9$, whereas the simplicity index of the CS represented by Diagram 20 is $2+2+7=11$ :
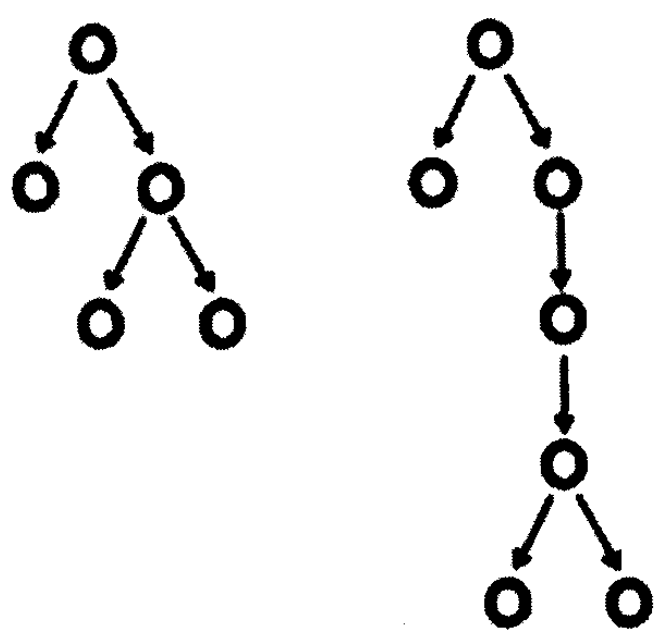

Diagram 19, Diagram 20. 
Assuming that two category systems adequately serve in respective ways the same explanatory purposes, we may find one system preferable to another on grounds among others of economy or complexity. Why do the same job with more categories or more complex interrelations among categories, indicated by breadth or spread of branchings plus total number of Nodes, than are strictly needed? Realistically, we may nevertheless be inclined by experience to expect that a less economical category system may have more than compensating virtues. It may articulate a more useful choice of further, more informatively subdivided subsumed categories. Only structurally complex category systems have more than Nodes at any given level of the system diagram. The more occurring collectively throughout the tree, the more complex the tree, even if overall it is more economical than alternatively structured category system trees. As appears in this comparison of previously seen category system diagrams:
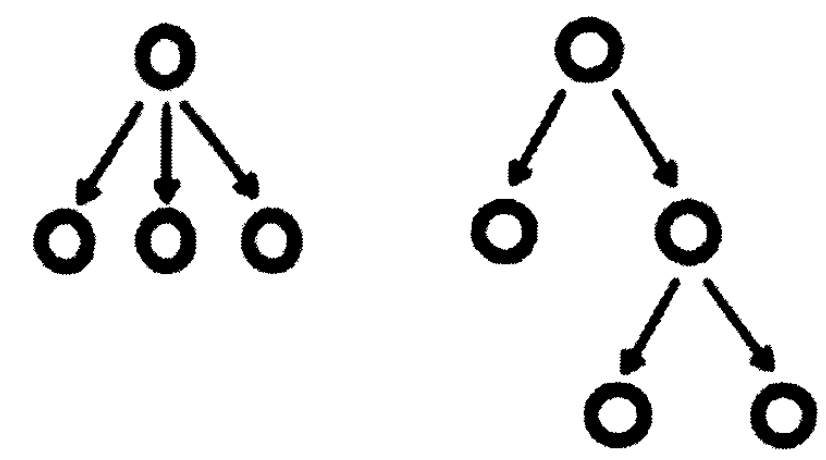

Diagram 10, Diagram 19.

Here we discover that the simplicity index of the category system corresponding to Diagram 10 is $3+4=7$, while that of Diagram 19 is $2+2+5=9$, and as such is structurally more complex than the category system represented by Diagram 19, according to the proposed criterion in (P3). With these concepts and criteria clarified and illustrated, we can say, finally, as a criterion for preference, on grounds of relative simplicity, by parity with previous comparative economy preference principle (P2):

$$
\text { (P4) } \forall C S_{m, n}\left[\operatorname{Simp}^{>}\left(C S_{m}, C S_{n}\right) \rightarrow s \operatorname{Pref}^{>}\left(C S_{m}, C S_{n}\right)\right]
$$

\section{CONFLICTING AND INCOMMENSURABLE PREFERENCE ORDERINGS}

What, now, to say, if $s \operatorname{Pref}^{>}\left(C S_{m}, C S_{n}\right) \wedge \neg e P r e f^{>}\left(C S_{m}, C S_{n}\right)$, or the reverse? Is there a natural order of precedence between these potentially conflicting preference factors in adjudicating between competing category systems? The formal criteria for category system preference presented here may not settle all such conflicts. It contributes to their resolution by making clear what is at stake in distinct and not always compatible preference grounds, and laying out the alternatives for choice as transparently as possible. Many applications might favor PPref $^{>}$over $e \mathrm{Pref}^{>}$, always on the assumption that explanatory burdens are equally adequately satisfied by competing category systems. 
The uninterestingly correct answer to this general question is that the comparison depends on the purpose to which the category system is supposed to be applied. What do we need the system for, what is it supposed to help explain, and how exactly do we intend to use it? All these factors are in keeping with the overarching conception of category system building as a purposive human cognitive activity that aspires to an accurate reflection of the metaphysical reality under categorical analysis. Would a category system's preference rating for economy versus simplicity trump its rating for the other? Discounting trivial reasons like tradition and culture, could it reasonably outweigh all or even any other considerations in making and epistemically, rationally, or philosophically justifying a category system preference? To venture this prioritization is only to enshrine the presumed fact that in most such applications we are more interested in what categories are included and how they are structured and fit together in the interrelations that a category system is charged with explaining, than we are in their simple differential numbers. Where questions of ontological economy are properly represented by a simple head count of category system categories without concern for their distinct internal subsumption structures, there we may find an adequate comparative preferability criterion in a purely economic comparison of the number of categories belonging to rival categories alone.

Always assuming comparable explanatory coverage by competing category systems, we should not overlook the possibility that two category systems might have identical cardinality of Nodes and a sum total of Nodes in a category system tree at every level bearing a cardinality of Nodes $\geq 2$, and hence that $\operatorname{Index}(\operatorname{Simp}(C S 1))=\operatorname{Index}(\operatorname{Simp}(C S 2))$, even though $(C S 1) \neq(C S 2)$. That is how simple, and equally, as it should be, how complex, the relevant category system is judged to be, on the basis of its branching tree formation plus its total number of Nodes. The identity of simplicity indexes for the two category systems in question can concur, schematically speaking, if the exact structure of Nodes and Links is preserved from one category system to another, although the contents of one or more Nodes are changed around with others originally situated at another Node. We have different category systems, for example, although both have precisely the same economy and simplicity (complexity) indexes, for example, if we switch Substance with Being in Brentano's reconstruction of Aristotle's branching category system represented in Diagram 4. The principle in (P3) does not, nor should it guarantee, that every different category system has a different comparative simplicity index, no more than (P2) guarantees that every different category system must always exemplify a different comparative economy index. When simplicity cancels out, economy and a host of other considerations are strengthened in their bid, and thereby given their first opportunity to influence, category system preference. Relative economy or simplicity (complexity) virtues, individually or in league, consequently, do not exhaust the parameters for preference among competing category systems. ${ }^{7}$

1. Roderick M. Chisholm, A Realistic Theory of Categories: An Essay on Ontology (Cambridge: Cambridge University Press, 1996), p. 3. 
2. Franz Brentano, On the Several Senses of Being in Aristotle, trans. of Von der mannigfachen Bedeutung des Seienden nach Aristoteles, (Freiburg im Breisgau: Herder'sche Verlagshandlung by Rolf George), (Berkeley: University of California Press, 1975), 115.

3. I offer this diagram in Dale Jacquette, "Brentano on Aristotle's Categories: First Philosophy and the Manifold Senses of Being," Revue roumaine de Philosophie 55: 2011, 192; reprinted in Franz Brentano's Metaphysics and Psychology: Upon the Sesquicentennial of Franz Brentano's Dissertation, edited by Ion Tanasescu (Bucharest: Zeta Books, 2012), 91.

4. Inspirational reading on Harry Beck's stylized map of London Underground lines and connections appears in Steven Heller and Veronique Vienne, 100 Ideas that Changed Graphic Design (London: Laurence King Publishing, 2012), 126-27. Design for Information: An Introduction to the Histories, Theories, and Best Practices Behind Effective Information Visualizations (Minneapolis: Rockport Publishers, 2013), especially pp. 9, 216-20. Although there are precedents, the idea of using branching tree diagrams to represent phy. logenetic evolutionary information category structures is often credited to Ernst Haeckel (1834-1919). See Haeckel's popular and widely disseminated illustrations to his, Generelle Morphologie der Organismen. Allgemeine Grundzüge der organisches Formen-Wissenschaft, mechanisch begründet durch die von Charles Darwin reformirte Descendenz-Theorie (Berlin: Druckk und Verlag von Geoerg Reimer, 1866).

5. Brentano, The Theory of Categories, trans. Roderick M. Chisholm and Norbert Guterman (The Hague: Martinus Nijhoff Publishers, 1981), 148. Asterisk note at bottom of the page attached to the diagram concludes with the parenthetical remark, "[Diagram and notes by A. Kastil]."

6. Among numerous sources, see especially Margaret J. Anderson, Carl Linnaeus: Father of Classification (Enslow Publishers, 2009); Paul Lawrence Fischer, Finding Order in Nature: The Naturalist Tradition from Linnaeus to E.O. Wilson (Baltimore: Johns Hopkins University Press, 2000); Wilfrid Blunt, Linneaus: The Compleat Naturalist, (Princeton: Princeton University Press, 2002). Linneaus's taxonomic category system is explained in general terms in application to plants in Linnaeus' Philosophia Botanica, trans. Stephen Fraser, (Oxford: Oxford University Press, 2005).

7. Thanks to Tina M. Jacquette for graphic design assistance in preparing category diagrams. 